
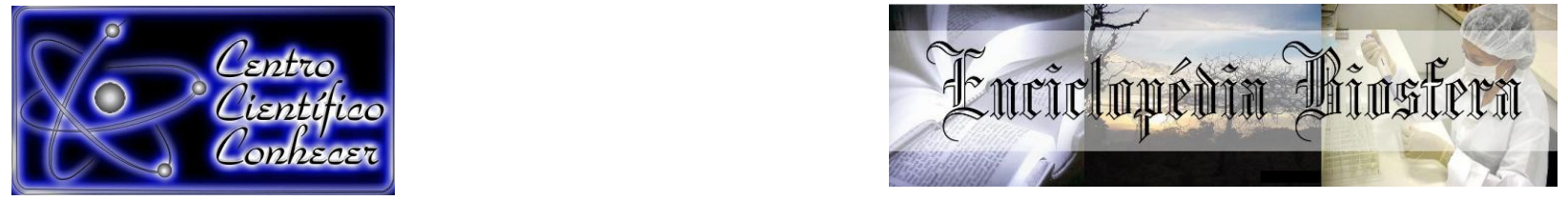

\title{
USO DE VEÍCULOS AÉREOS NÃO TRIPULADOS (VANT) PARA MENSURAÇÃO DE PROCESSOS FLORESTAIS
}

\author{
Marcos Fidêncio de Oliveira Sobrinho ${ }^{1}$, Ana Paula Dalla Corte ${ }^{2}$ Bruna Nascimento de \\ Vasconcellos ${ }^{3}$, Carlos Roberto Sanquetta ${ }^{2}$, Franciel Eduardo Rex ${ }^{4}$ \\ 1. Engenheiro Florestal - Especialista em Manejo Florestal de Precisão - UFPR; \\ 2. Dr., Professor do Curso de Engenharia Florestal - UFPR; \\ 3. Doutoranda em Engenharia Florestal - UFPR; \\ ${ }^{4}$. Mestrando em Engenharia Florestal - UFPR; \\ Centro BIOFIX de Pesquisa em Biomassa e Sequestro de Carbono Universidade \\ Federal do Paraná - UFPR E-mail de contato: marcos_engeflorestal@hotmail.com
}

Recebido em: 06/04/2018 - Aprovado em: 10/06/2018 - Publicado em: 20/06/2018 DOI: 10.18677/EnciBio_2018A80

\begin{abstract}
RESUMO
A utilização de veículos aéreos não tripulados (VANT) tem se destacado como uma ferramenta de grande potencial para obtenção de informações em diversas áreas do conhecimento. Nesse sentido, o objetivo desta pesquisa foi avaliar o desempenho de imagens coletadas com VANT para automatizar a geração de informações, tendo em vista aplicações de silvicultura de precisão, tais como: avaliação de sobrevivência, quantificação do estoque de madeira em campo e quantificação dos resíduos de processos florestais. O desenvolvimento desta pesquisa foi realizado em três fazendas, localizadas no Centro-Oeste do estado de São Paulo. As avaliações realizadas foram de: sobrevivência aos 15 dias após o plantio de Eucalyptus grandis e Eucalyptus urograndis, quantificação do estoque de madeira em campo, e quantificação dos resíduos de processo em campo, empregando o VANT modelo X800 Geo. Os resultados obtidos com o VANT revelaram-se satisfatórios. Quanto à sobrevivência, o VANT apresentou o melhor resultado de correspondência com a recomendação, apresentando $96,86 \%$ de sobrevivência. Quanto ao estoque de madeira em campo $\left(\mathrm{m}^{3}\right)$, ocorreu uma variação de $0,67 \%$ a $3,57 \%$ em relação ao estimado pelo método convencional. Os resíduos florestais apresentaram um volume médio por parcela de $0,64 \mathrm{~m}^{3}$, de modo que, o volume total foi de $9,09 \mathrm{~m}^{3} \mathrm{ha}^{-1}$.
\end{abstract}

PALAVRAS-CHAVE: Drone, ortomosaico, precisão.

\section{USE OF UNMANNED AERIAL VEHICLES (UAV) FOR MEASUREMENT OF FOREST PROCESSES}

\begin{abstract}
The use of unmanned aerial vehicles (UAV) has stood out as a tool with great potential for obtaining information in several areas of knowledge. In this sense, the goal of this research was to evaluate the performance of images collected with VANT to automate the generation of information for precision silviculture applications, such as: for the evaluation of survival, quantification of wood stock in the field and quantification of forest process residues. The development of this research was carried out in three farms, located in the Center-West of the state of São Paulo. The evaluations were: survival at 15 days after planting Eucalyptus grandis and ENCICLOPÉDIA BIOSFERA, Centro Científico Conhecer - Goiânia, v.15 n.27; p. 117 2018
\end{abstract}


Eucalyptus urograndis, quantification of wood stock in the field and quantification of process residues in the field, using the UAV model X800 Geo. The results obtained with the UAV were satisfactory. Regarding survival, the UAV presented the best matching result with the recommendation, presenting $96.86 \%$ survival. As for wood stock in the field $\left(\mathrm{m}^{3}\right)$, there was a change from $0.67 \%$ to $3.57 \%$ in relation to that estimated by the conventional method. The forest residues had an average volume per plot of $0.64 \mathrm{~m}^{3}$, so that the total volume was $9.09 \mathrm{~m}^{3}$ ha-1.

KEYWORDS: Drone, orthophoto, precision.

\section{INTRODUÇÃO}

Os recentes esforços em pesquisas mostraram que, os dados tridimensionais (3D) gerados a partir de imagens coletadas usando veículos aéreos não tripulados (VANT) são fontes viáveis de dados para apoiar práticas florestais (DANDOIS; ELLIS, 2013; LISEIN et al., 2013). Desde seu advento, estes veículos têm oferecido oportunidades promissoras para o monitoramento de fenômenos ambientais (ANDERSON; GASTON, 2013) e tem se destacado particularmente na última década, se consolidando como uma nova forma de obtenção de parâmetros suborbitários de alta qualidade espacial, espectral e multissensorial (ALMEIDA, 2010; COLOMINA; MOLINA, 2014; DIAS; ROSSA, 2015).

A utilização desta tecnologia quando comparado com as técnicas de sensoriamento remoto obtidas via satélite, aérea e/ou métodos de aquisição de imagens de alta resolução, aparece como alternativa mais viável (GRAÇA, 2017). Estes veículos se apresentam superiores, pois podem voar em baixas altitudes e em velocidade lenta, permitindo a obtenção de alta resolução espacial de imagens (KOH; WICH, 2012; WHITEHEAD; HUGENHOLTZ, 2014).

Outro destaque na utilização de VANT está relacionado a frequência das medidas ao longo do tempo, ou seja, a resolução temporal (JAAKKOLA et al., 2010; DANDOIS et al., 2015). Aumentando a resolução temporal, os VANT oferecem oportunidades únicas para monitorar mudanças nos recursos florestais ao longo do tempo (WHITEHEAD et al., 2014).

$\mathrm{Na}$ literatura já existe uma série de estudos que demonstraram a eficiência da utilização de imagens de VANT, como por exemplo, no mapeamento de espécies invasoras (MICHEZ et al., 2016), no planejamento de uso e cobertura do solo (CANDIDO et al., 2015) no monitoramento da variação da estrutura da floresta e na análise do padrão espacial (INOUE et al., 2014) bem como, na alteração do uso do solo (PANEQUE-GÁLVEZ, 2014).

Porém, são escassas as pesquisas que apresentam metodologias e resultados promissores utilizando imagens de VANT nos processos de silvicultura de precisão. De acordo com Medeiros (2007), no Brasil as pesquisas ainda são recentes, e os trabalhos com VANT têm no escopo as aplicações civis, como vigilância policial de áreas urbanas e de fronteira, inspeções de linhas de transmissão de energia, monitoramento, atividades de áreas agrícolas, acompanhamento de safra, controle de pragas e de queimada.

Recentemente, estudo dirigido por Ruza et al. (2017) revelou-se muito promissor para o meio florestal. Os autores testaram o desempenho da técnica de redes neurais artificiais, em ortofotos obtidas por VANT, para a detecção e extração automática de mudas de árvores, visando subsidiar e/ou substituir o inventário florestal de sobrevivência em campo. Estudos neste escopo impulsionam o desenvolvimento de novos estudos, pois demonstram que a utilização desta tecnologia se revela como um instrumento potencial que pode subsidiar e fomentar a 
obtenção de informações, promovendo melhorias e avanços para a pesquisa e para o mercado florestal.

Nesse sentido, o objetivo deste trabalho foi testar o desempenho do uso de imagens coletadas com VANT para a automação na geração de informações, visando aplicações de manejo e silvicultura dos plantios florestais, como: para a avaliação de sobrevivência aos 15 dias após o plantio, quantificação do estoque de madeira em campo e quantificação dos resíduos de processos florestais.

\section{MATERIAL E MÉTODOS}

\section{Caracterização experimental}

Esta pesquisa foi desenvolvida em três fazendas da região Centro-Oeste do estado de São Paulo. Na tabela 1 são expostas as informações referentes as condições de cada fazenda.

TABELA 1. Especificações das fazendas $A, B$ e $C$ utilizadas para as avaliações de sobrevivência, estoque de madeira em campo e resíduo de processo.

\begin{tabular}{cccccc}
\hline Fazenda & Talhão & Área (ha) & Espécie & Data de Plantio & $\begin{array}{c}\text { Espaçamento } \\
(\mathbf{m} \times \mathbf{~ m})\end{array}$ \\
\hline A & 01102 & 11,21 & E. grandis & $02 / 04 / 2008$ & $3,0 \times 3,0$ \\
B & 01702 & 11,65 & E. grandis & $26 / 02 / 2009$ & $3,8 \times 2,1$ \\
B & 01703 & 11,65 & E. urograndis & $29 / 07 / 2016$ & $3,8 \times 2,1$ \\
C & 05102 & 16,34 & E. grandis & $26 / 02 / 2009$ & $3,8 \times 2,1$ \\
\hline
\end{tabular}

FONTE: Os autores.

As coletas de dados em campo foram realizadas em talhões, entre os meses de junho a setembro de 2016. Utilizou-se o VANT modelo X800 Geo, produzido pela empresa XFly Brasil com 1,18 m de envergadura e 2,5 kg, controlado remotamente.

Foram definidos diferentes valores de GSD (Ground Sample Distance), uma vez que, esta é uma definição muito importante de um projeto fotogramétrico, pois representa o tamanho real em unidades do terreno, que um determinado pixel representa em função da resolução de uma imagem (BRITO; COELHO, 2002). Para as avaliações realizadas utilizou-se:

- Avaliação de sobrevivência: GSD 2,0 a 3,0 cm;

- Quantificação dos estoques de madeira em campo: GSD 3,0 a 5,0 cm;

- Quantificação dos resíduos de processo em campo: GSD 3,0 a 5,0 cm.

O trabalho foi dividido em três etapas, sendo: avaliação da sobrevivência aos 15 dias de plantio, quantificação do estoque de madeira no campo, quantificação dos resíduos dos processos florestais. Na Figura 1 é possível visualizar a sequência metodologia utilizada. 

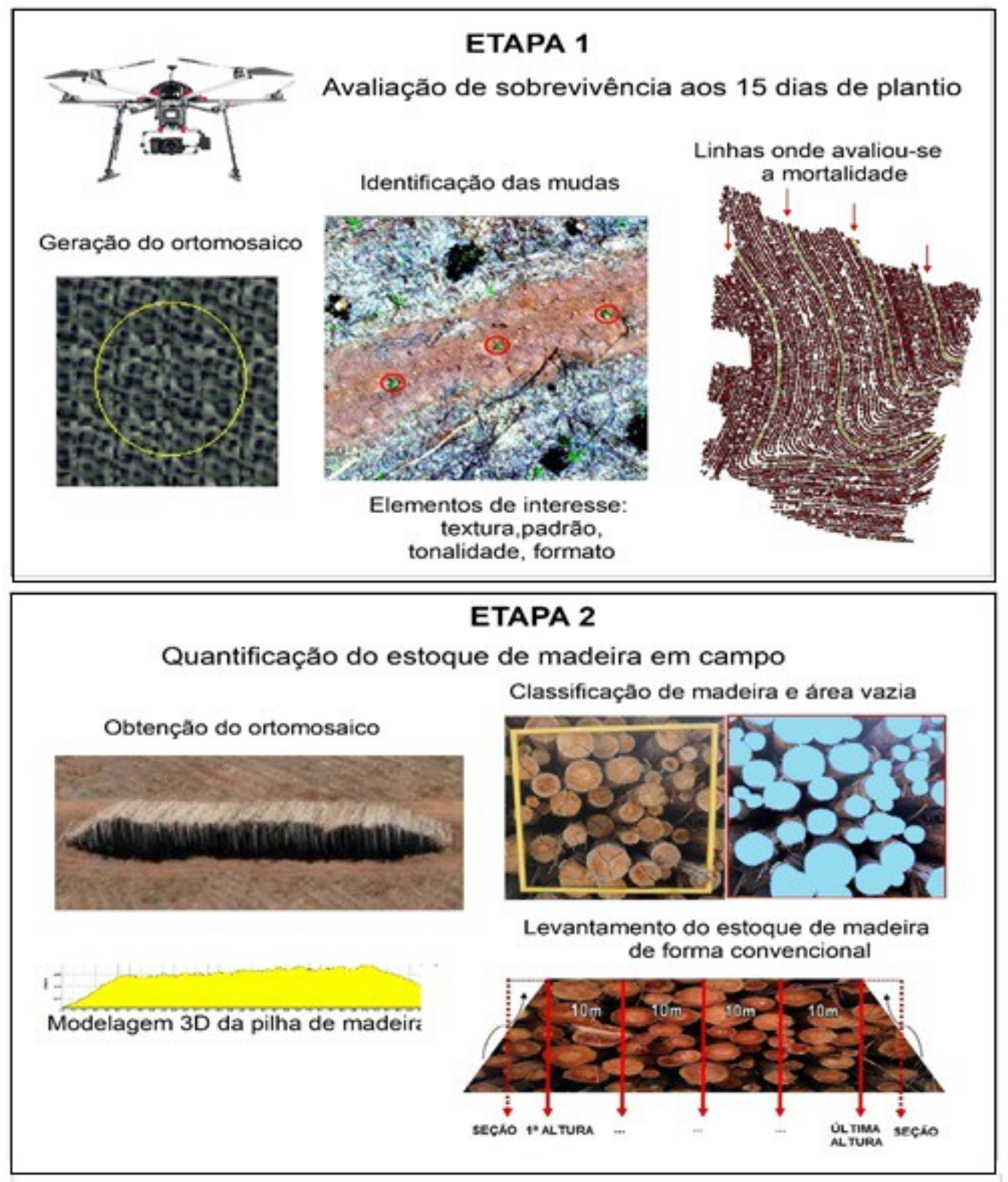

\section{ETAPA 3}

Quantificaçăo dos residuos de processos florestais
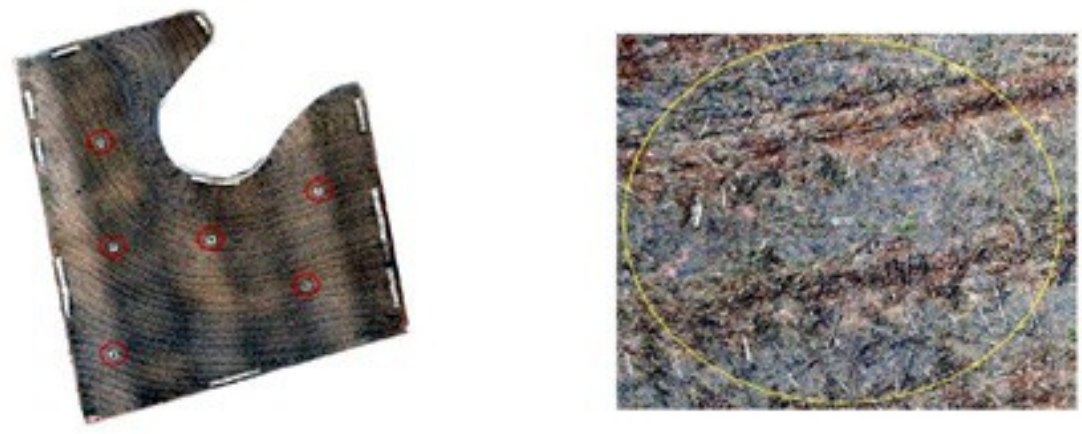

Implantaçăo de parcelas aleatórias para cubagem

Implantaçăo de parcelas sistemáticas para estimar o volume de residuos

FIGURA 1 - Etapas da mensuração de processos florestais utilizando VANT. FONTE: Os autores 


\section{Avaliação de sobrevivência aos 15 dias após o plantio}

Inicialmente gerou-se o mosaico de ortofoto, utilizado para modelagem 3D e mapeamento. O processamento das imagens foi realizado com o auxílio de algoritmos computacionais, de forma automatizada, para grandes lotes de imagens, já objetivando a aplicação em escala operacional. A etapa inicial consistiu em uma fase de pré-processamento das imagens, com operações de equalização, ajuste de brilho, contraste, ajuste por histograma, entre outros.

Logo, foram aplicadas técnicas de processamento de imagens específicas para o tema analisado, sendo: segmentação, análise de forma e área de objetos. A metodologia empregada na identificação de alvos foi baseada na interpretação dos elementos que o caracterizam, sendo: formato, tamanho, tonalidade, padrão, textura e localização. A combinação desses elementos e processamento através do PhotoScan, QGIS e ArcGIS, possibilitou discernir um alvo de interesse de um ruído de imagem.

Para este estudo, foram consideradas apenas as linhas inteiras dos plantios, sendo desconsideradas as linhas iniciais por serem curtas e também as linhas que não possuíam continuação ao longo de todo talhão, pois não possuem representatividade. A partir da primeira linha, onde iniciou-se a avaliação, ao final da contagem de plantas na linha, utilizou-se um intervalo de 15 linhas e iniciou-se uma nova contagem na $16^{a}$ linha de plantio.

\section{Quantificação do estoque de madeira em campo}

O software utilizado foi o PhotoScan para modelagem 3D das pilhas de madeira, e assim, obtenção do volume estéreo (st). Após a quantificação da variável, foi realizado o levantamento do fator de empilhamento nas pilhas de madeira amostradas em campo para obtenção do volume em metros cúbicos $\left(\mathrm{m}^{3}\right)$. O princípio de identificação foi realizado em um alvo fixo de $1 \mathrm{~m}^{2}$, onde o sistema realizou a varredura das imagens (pixel a pixel), agrupando pixels que possuíam as mesmas características e classificando-os como madeira e área vazia. $O$ fator de conversão de volume (st para $\mathrm{m}^{3}$ ) foi obtido pela relação de área de madeira presente em $1 \mathrm{~m}^{2}$ do gabarito.

Para o levantamento do estoque de madeira em campo, foi realizada a medição das pilhas de madeira, onde considerou-se a primeira altura a partir do ponto em que a mesma segue ao longo do perfil da pilha de madeira. A cada 10 metros foi coletada uma nova altura até o final do perfil da pilha, as "pontas" foram seccionadas representando 0 encaixe formando um retângulo. Mediu-se 0 comprimento da pilha de madeira com fita métrica de $50 \mathrm{~m}$.

Em escritório, foi feito a média das alturas, multiplicou-se pelo comprimento da tora (valor padrão) 6,20m e este resultado multiplicou-se pelo comprimento da pilha de madeira, obtendo assim o volume estéreo (st). Conforme pode ser visualizado na Equação 1:

Vol $_{\text {st }}=\dot{X}_{\text {alt }} \times 6,20$ (valor padrão) $\times \mathrm{L}$

Onde:

$\mathrm{Vol}_{\mathrm{st}}=$ volume estéreo;

$\dot{X}_{\text {alt }}=$ média das alturas;

$6,20 \mathrm{~m}=$ comprimento do traçamento das toras;

$\mathrm{L}=$ Comprimento da pilha. 
Para obtenção do volume em metros cúbicos $\left(\mathrm{m}^{3}\right)$ determinou-se o fator de empilhamento, sendo a metodologia empregada semelhante com a utilizada pelo VANT. Colocou-se um gabarito de $1 \mathrm{~m}^{2}$ na pilha de madeira e mediu-se o diâmetro de todas as toras dentro do esquadro. Esses valores determinaram o fator para a pilha de madeira ponderando as amostras coletadas pelo volume estéreo. Este fator foi utilizado para a conversão do volume em metros cúbicos $\left(\mathrm{m}^{3}\right)$.

Para análise comparativa considerou-se os dados de madeira posto na fábrica. O primeiro dado foi o volume estéreo, obtido através da coleta da altura da carga já no caminhão; comprimento das toras, através do controle de qualidade do processo de colheita e largura do caminhão, que foram lançados no sistema ERP (Enterprise Resource Planning) e retornaram o valor em estéreo. Outro dado, foi o peso em toneladas ( $\mathrm{t}$ ), o caminhão foi pesado na balança ao sair da fábrica e ao retornar carregado pesado novamente, obtendo o volume de carga em toneladas.

\section{Quantificação dos resíduos de processos florestais}

A quantificação dos resíduos deixados pelos processos florestais, é uma informação muito almejada pelas empresas, pois sua utilização apresenta um grande potencial para a geração de subprodutos, tais como energia, briquetes ou pellets para posterior combustão (WIECHETECK, 2009).

Com o uso do VANT foi realizada a quantificação desses resíduos. Para tal, inicialmente foram instaladas seis parcelas aleatórias de aproximadamente $703 \mathrm{~m}^{2}$ para cubagem rigorosa dos resíduos do processo de remoção de forwarder. Essa cubagem auxiliou no ajuste da equação volumétrica com base nas variáveis de diâmetro, comprimento e superfície para o modelo de colheita harvester + forwarder sistema toras curtas, tendo como base um modelo exponencial (ajustado pela empresa). Logo, para o processamento das amostras e quantificação dos resíduos, foram instaladas 19 parcelas sistemáticas de aproximadamente $703 \mathrm{~m}^{2}$. Após o processamento do algoritmo ajustado ao modelo, obteve-se o volume de resíduos por parcela.

\section{RESULTADOS E DISCUSSÃO}

\section{Avaliação de sobrevivência aos 15 dias após o plantio}

$\mathrm{Na}$ Tabela 2 é possível visualizar o total de plantas contabilizadas por meio de censo e amostragem obtidas com o VANT, e ainda, a contabilização pelo método convencional, e o que seria recomendado de acordo com o "stand" ou (densidade de plantio) utilizado pela empresa.

TABELA 2. Avaliação comparativa de sobrevivência aos 15 dias após o plantio.

\begin{tabular}{lccc}
\hline & $\begin{array}{c}\text { Total de } \\
\text { plantas }\end{array}$ & $\begin{array}{c}\text { Stand } \\
\left(\text { mudas.ha }^{-1}\right)\end{array}$ & $\begin{array}{c}\text { Sobrevivência } \\
(\%)\end{array}$ \\
\hline Censo & 12.149 & 1.204 & 94,29 \\
Amostragem & 12.480 & 1.237 & 96,86 \\
Convencional & 12.259 & 1.215 & 95,14 \\
Recomendado & 12.885 & 1.277 & 100,00 \\
\hline
\end{tabular}

\section{FONTE: Os autores}

De acordo com o resultado da Tabela 2, o "stand" recomendado é de 1.277 mudas.ha ${ }^{-1}$, para o caso de $100 \%$ de sobrevivência. Por ser uma área de reforma 
florestal, é realizado o plantio entre as cepas do plantio anterior, interferindo no espaçamento. Porém, quando há um espaçamento maior entre linhas do que o padrão utilizado, adensa-se o espaçamento entre plantas para manter o "stand".

A amostragem realizada através de VANT apresentou o melhor resultado de equivalência com a recomendação. Acredita-se que o método de amostragem seja menos tendencioso que o censo. Pois principalmente nas áreas de reforma, ocorre os realinhamentos ou plantio entre cepas e o censo não faz essa leitura do reajuste do espaçamento. Relacionando essas informações com as abordagens realizadas no inventário florestal, tem-se que o êxito na obtenção de informações precisas está intimamente relacionado aos processos e métodos de amostragem a serem adotados, assim como à intensidade amostral adotada (UBIALLI et al., 2009; CORTE et al., 2013).

Estudo recente dirigido por Puliti et al. (2017) consideram que o uso de dados obtidos por meio de VANT's são eficazes no inventário florestal, pois a combinação de dados espaciais com dados de campo aumenta a precisão das estimativas, e oferece uma alternativa econômica para avaliação de recursos florestais em larga escala. Chianucci et al. (2016) consideram que as imagens obtidas por meio de VANT's são eficientes, pois permitem obter estimativas rápidas e baratas, além do que, as imagens de alta resolução permitem realizar análises de monitoramento de "stands" florestais e de rotina em tempo real, sendo considerado como grande potencial no inventario florestal. A seguir a Figura 2 ilustra imagens do voo de identificação e a classificação das mudas. As mudas foram convertidas para o formato vetorial, e contabilizadas
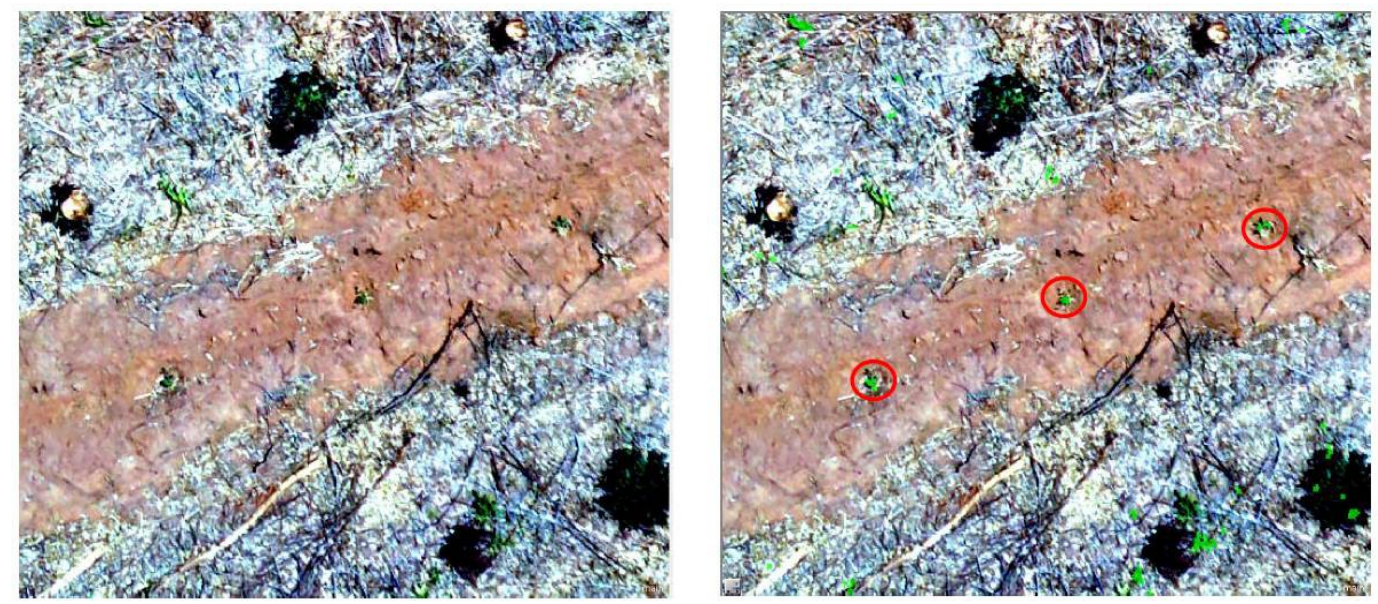

FIGURA 2. Voo de identificação (esquerda) e classificação das mudas (direita).

FONTE: Os autores.

Na Figura 2 é possível visualizar que a obtenção e o processamento de imagens de VANT's vem se revelando como um instrumento muito importante para o apoio em obtenção de informações, neste caso, a identificação e a classificação das mudas apresentaram resultados nítidos e satisfatórios. Além disso, foi possível identificar a localização geográfica das áreas que apresentaram mortalidade. De acordo com Vergani (2015) informações de sobrevivência são consideradas como suporte à decisão para ações de mitigação para os casos de baixa sobrevivência, principalmente no que se refere à compensação de fustes e plantio de adensamento. 
Ruza et al. (2017) realizaram o inventário de sobrevivência de povoamento de Eucalyptus com uso de redes neurais artificiais em fotografias obtidas por VANT e alcançaram resultados satisfatórios. Os autores consideram ainda, que o uso desta tecnologia para as grandes empresas florestais é vantajoso, quando comparados as imagens de satélites.

\section{Quantificação do estoque de madeira em campo}

Os resultados do estoque de madeira em campo obtidos através da medição das pilhas de madeira e levantamento com VANT foram comparados com base na informação de madeira posto fábrica. As Tabelas 3 e 4 apresentam os resultados obtidos para as fazendas $\mathrm{A}$ e $\mathrm{B}$, respectivamente.

TABELA 3. Comparativo dos volumes de madeira nas fazendas A e B.

\begin{tabular}{|c|c|c|c|c|c|c|c|c|c|}
\hline & Tipo & $\begin{array}{l}\text { Volume } \\
\text { (st) }\end{array}$ & $\begin{array}{c}\text { Variação } \\
\text { st }(\%)\end{array}$ & $\begin{array}{l}\text { Fator } \\
\mathrm{st} / \mathrm{m}^{3}\end{array}$ & $\begin{array}{c}\text { Volume } \\
\qquad\left(\mathrm{m}^{3}\right)\end{array}$ & $\begin{array}{c}\text { Variação } \\
\text { m }^{3}(\%)\end{array}$ & $\begin{array}{c}\text { Fator } \\
\text { st/t }\end{array}$ & $\begin{array}{l}\text { Volume } \\
\text { (t) }\end{array}$ & $\begin{array}{c}\text { Variação } t \\
(\%)\end{array}$ \\
\hline \multirow{3}{*}{ 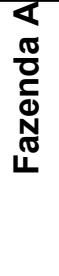 } & $\begin{array}{l}\text { Medição de } \\
\text { campo }\end{array}$ & $6.012,33$ & 94,44 & 1,65 & $3.643,84$ & 91,58 & 0,43 & $2.585,30$ & 80,72 \\
\hline & VANT & $6.166,57$ & 96,86 & 1,68 & $3.670,58$ & 92,25 & 0,43 & $2.774,96$ & 86,64 \\
\hline & Fábrica & $6.366,22$ & 100,00 & - & $3.978,89$ & 100,00 & - & $3.202,89$ & 100,00 \\
\hline \multirow{3}{*}{$\begin{array}{l}0 \\
\frac{\pi}{0} \\
\frac{0}{0} \\
\mathbb{N} \\
\mathbb{N} \\
\text { L }\end{array}$} & $\begin{array}{l}\text { Medição de } \\
\text { campo }\end{array}$ & $5.844,36$ & 97,49 & 1,60 & $3.643,95$ & 111,01 & 0,43 & $2.513,07$ & 111,01 \\
\hline & VANT & $6.099,67$ & 101,75 & 1,73 & $3.527,02$ & 107,44 & 0,43 & $2.622,86$ & 115,86 \\
\hline & Fábrica & $5.994,61$ & 100,00 & - & $3.282,67$ & 100,00 & - & $2.263,91$ & 100,00 \\
\hline
\end{tabular}

\section{FONTE: Os autores}

De acordo a Tabela 3, pode-se observar que o volume (st) da Fazenda A apresenta uma variação de $2,42 \%$ entre o levantamento com VANT e a medição de campo realizado pela empresa. Entretanto, ao analisar o volume $\left(\mathrm{m}^{3}\right)$ a diferença reduziu para $0,67 \%$. Ao comparar os levantamentos com o volume estéreo posto fábrica, é subestimado em 3,14\% e 5,56\% o volume de madeira. Quando o volume é convertido de metro estéreo para metros cúbicos, essa diferença passa a ser $7,75 \%$ e $8,42 \%$, respectivamente.

Pode-se observar também que ao converter o volume estéreo para tonelada, a variação entre os resultados dos levantamentos comparados com o de fábrica aumentam. Porém, o fator utilizado para conversão (st/t), é um valor padrão adotado pela empresa. Como a densidade, teor de umidade da madeira e outros fatores interferem na pesagem da madeira, o erro pode ser considerado como um fator com valor fixo.

Em relação a Fazenda $B$, observa-se que no volume estéreo existe uma variação de $4,26 \%$ entre o levantamento com VANT e a medição de campo convencional realizada pela empresa. Entretanto, ao analisar o volume $\mathrm{m}^{3}$ a diferença reduziu para $3,57 \%$.

Ao comparar com o volume em estéreo posto fábrica, a medição de campo subestimou em $2,51 \%$ e o VANT superestimou em $1,75 \%$ o volume de madeira. Quando se converte de estéreo para metros cúbicos há uma superestimava de $11,01 \%$ e $7,44 \%$, respectivamente. Pode-se observar também que ao converter o ENCICLOPÉDIA BIOSFERA, Centro Científico Conhecer - Goiânia, v.15 n.27; p. 124 2018 
volume estéreo para tonelada, a variação entre os resultados dos levantamentos comparados com o de fábrica aumentam.

Autores como Figueiredo et al. (2016) estimaram o volume de madeira em pátios de estocagem de toras por meio de câmeras RGB instaladas em VANT. Os erros de estimativa volumétrica variaram de 0,58 a $19,63 \%$, variando conforme os diferentes planos de voo testados. Os voos com $30 \mathrm{~m}$ apresentaram valores aceitáveis para uma atividade de averiguação de volume estocado em pátio.

Kung et al. (2011) consideram que ao estimar volume por meio de VANT, os maiores erros estão associados ao plano de voo, de modo que, quanto mais alto o voo, maior será o erro. Porém, os equipamentos e a capacidade de processamento de dados têm evoluído significativamente, de modo que, obtém-se uma melhor acurácia do produto final (ROKHMANA, 2015).

Dos resultados apresentados, a única medição realizada para os três fatores analisados é o volume em estéreo. Com base nesse fator, observa-se que o levantamento de VANT é o resultado que mais se aproxima do posto fábrica, logo o que melhor representa o valor real. Os demais fatores analisados são obtidos através de fatores de transformação o que pode acarretar em erros devido à influência de variáveis não analisadas.

\section{Quantificação dos resíduos de processos florestais}

Para quantificação dos resíduos de processos florestais, foi considerada apenas as toras de madeiras descascadas. As quais foram agrupadas por classe de comprimento, e estratificadas como resíduo de processo do harvester as toras com comprimento menor que 3 metros, e resíduo de processo do forwarder toras maiores que 3 metros. Obteve-se um volume médio por parcelas de $0,64 \mathrm{~m}^{3}$. Dessa forma, o volume total foi de $9,09 \mathrm{~m}^{3}$.ha ${ }^{-1}$ (Figura 03).

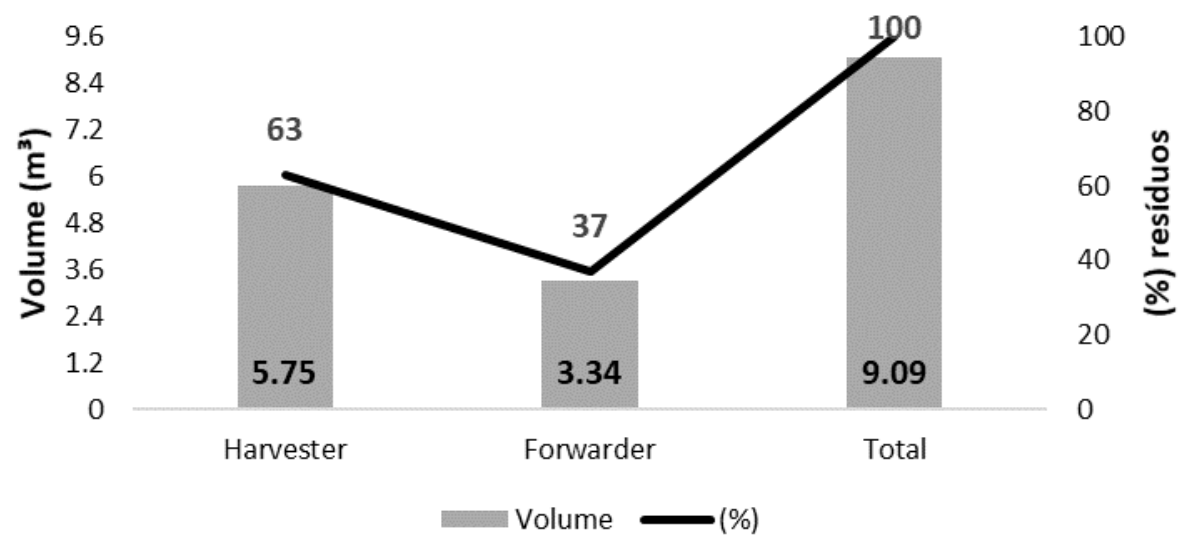

FIGURA 3. Estratificação dos resíduos de processos florestais. FONTE: Os Autores.

Através do resultado apresentado pôde-se observar que as principais questões são as falhas de processo. Os resíduos provenientes de harvester são toras processadas que não foram previamente dimensionadas e ao serem tracionadas, ficaram com comprimento inferior ao do processo de remoção.

As falhas do processo de remoção consistem no excesso do enchimento do compartimento de carga do forwarder, causando a queda de toras ao longo do 
deslocamento até a pilha e também a coleta das toras que não preenchem a garra no momento da coleta e são deixadas para trás.

Baggio e Carpanezzi (1995) quantificaram os resíduos florestais em Bracatingais no município de Curitiba e concluíram que a quantidade média de lenha abandonada depois da exploração equivale a $24 \%$ do peso total dos resíduos florestais, representando $16,5 \%$ da produtividade média dos talhões amostrados. $\mathrm{Na}$ Figura 4 é possível visualizar os resíduos identificados por meio do VANT, distribuídos em parcelas sistemáticas.
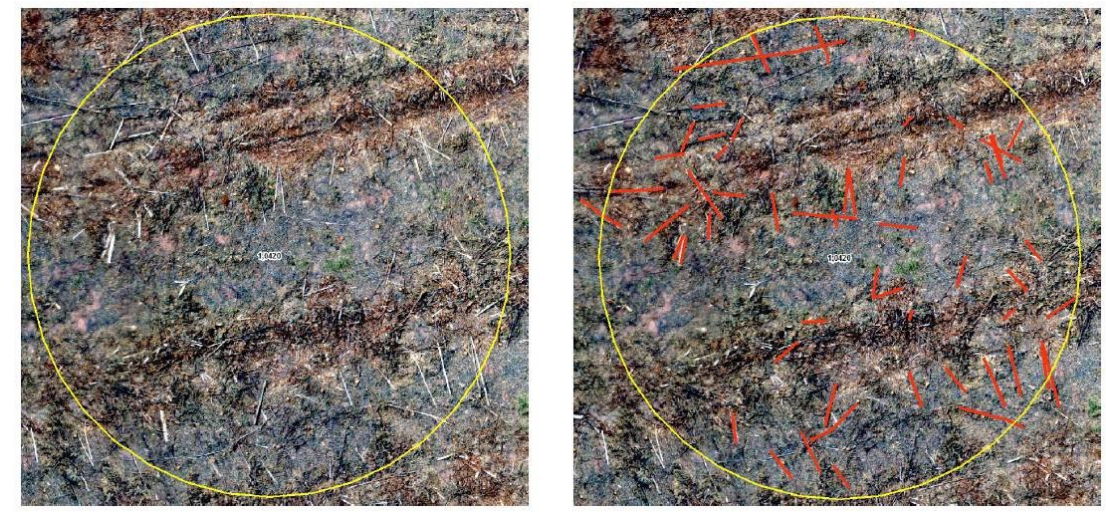

FIGURA 4. Parcelas sistemáticas para quantificação do resíduo. FONTE: Os Autores

De acordo com Zhang et al. (2016), o uso de VANT apresenta uma grande vantagem em relação as demais técnicas de sensoriamento remoto, pois podem voar em baixas altitudes e em velocidades lentas, de modo que, é possível obter imagens com uma alta resolução espacial (1 a $20 \mathrm{~cm}$ ). Além do mais, a combinação de baixa resolução espectral e alta resolução espacial das imagens coletadas com VANT propiciam novas abordagens no setor florestal, bem como a detecção de copas, quantificação de madeira e dos resíduos florestais (HUNG, 2012).

\section{CONCLUSÃO}

Os resultados obtidos através do VANT foram satisfatórios para a avaliação de sobrevivência aos 15 dias após o plantio, quando comparados com o método convencional. $\mathrm{O}$ uso deste equipamento permitiu, além da avaliação quantitativa, a avaliação geográfica e especializada do problema, tornando possível a tomada de decisão e a intervenção de forma eficaz e precisa.

Para a quantificação do estoque de madeira em campo, os resultados obtidos (volume estéreo) apresentaram baixa variabilidade com relação ao volume posto fábrica. Para a determinação do volume das pilhas de madeira e o fator de empilhamento das mesmas, os resultados, tanto quantitativos quanto de rendimento, foram superiores quando comparados ao método convencional de medição.

Para a quantificação dos resíduos de processos florestais, o uso do VANT se mostrou eficaz na classificação das classes diamétricas, podendo determinar qual dos processos podem contribuir para a geração destes resíduos. Para a determinação do volume dos mesmos também se obteve resultados satisfatórios e condizentes com a cubagem realizada em campo.

De forma geral, o uso do VANT apresentou resultados e rendimentos melhores que o método convencional de medição para os processos. Com esta abordagem é possível obter importantes resultados importantes para determinação de planos de ação em casos em que a produtividade possa ser comprometida, ENCICLOPÉDIA BIOSFERA, Centro Científico Conhecer - Goiânia, v.15 n.27; p. 126 2018 
reduzindo eventuais problemas de produção de madeira e abastecimento das fábricas consumidoras.

\section{REFERÊNCIAS}

ALMEIDA, J. Normas de Segurança para Implantação dos VANT Civis no Espaço Aéreo Brasileiro: uma nova abordagem. Conexão SIPAER, v. 2, n. 1, 2010.

ANDERSON, K.; GASTON, K. J. Lightweight unmanned aerial vehicles will revolutionize spatial ecology. Frontiers in Ecology and the Environment, v. 11, n. 3, p. 138-146, 2013. https://doi.org/10.1890/120150

BAGGIO, A. J.; CARPANEZZI, A. A. Quantificação dos resíduos florestais em Bracatingais na região metropolitana de Curitiba-PR. Boletim de Pesquisa Florestal, v.30, p.51-66, 1995.

BRITO, J. N.; COELHO, L. Fotogrametria Digital. Instituto Militar de Engenharia. $1^{\text {a }}$ ed. Rio de Janeiro, Brasil:. 212 p, 2002.

CHIANUCCI, F.; DISPERATI, L.; GUZZI, D.; BIANCHINI, D.; NARDINO, V.; LASTRI, C.; RINDINELLA, A.; CORONA, P. Estimation of canopy attributes in beech forests using true colour digital images from a small fixed-wing UAV. International Journal of Applied Earth Observation and Geoinformation, v.47, p.60-68, 2016. https://doi.org/10.1016/j.jag.2015.12.005

COLOMINA, I.; MOLINA, P. Unmanned aerial systems for photogrammetry and remote sensing: A review. ISPRS Journal of Photogrammetry and Remote Sensing, 92, 79-97, 2014. https://doi.org/10.1016/j.isprsjprs.2014.02.013

CORTE, A. P. D.; SANQUETTA, C. R.; FIGUEIREDO FILHO,A.;PEREIRA, T. K.; BEHLING, A. Desempenho de métodos e processos de amostragem para avaliação de diversidade em Floresta Ombrófila Mista. Floresta, v. 43, n. 4, p. 579-582, 2013. http://dx.doi.org/10.5380/rf.v43i4.30526

DANDOIS, J.P. ELLIS, E.C. High spatial resolution three-dimensional mapping of vegetation spectral dynamics using computer vision. Remote Sensing of Environment, v. 136, p. 259-276, 2013. https://doi.org/10.1016/j.rse.2013.04.005

DANDOIS, J.P.; OLANO, M; ELLIS, E.C. Optimal altitude, overlap, and weather conditions for computer vision UAV estimates of forest structure. Remote Sensing, v. 7 , n. 10 , p. $13895-13920,2015$. doi:10.3390/rs71013895

DIAS, H. G.; ROSSA, A. A. Considerações Sobre O Emprego De Veículos Aéreos Não Tripulados (VANT) Armados À Luz Do Direito Internacional Dos Conflitos Armados Considerations about the use of armed Unmanned Aerial Vehicles (UAVs) as seen through the light of the International Law. Coleção Meira Mattos-Revista das Ciências Militares, v. 9, n. 34, p. 189-200/201-212, 2015.

FIGUEIREDO, E.O.; OLIVEIRA, M.V.N.; LOCKS, C.J.; PAPA, D.A. Estimativa do volume de madeira em pátios de estocagem de toras pr meio de câmeras de RGB 
instaladas em aeronaves remotamente pilotadas. Embrapa Acre-Boletim de Pesquisa e Desenvolvimento (INFOTECA-E), 39p., 2016.

GRAÇA, N.L.S.D.S., MITISHITA, E. A, \& GONÇALVES, J. E. Use of UAV platform as an autonomous tool for estimating expansion on invaded agricultural land. Boletim de Ciências Geodésicas, v. 23, n. 3, p. 509-519, 2017. http://dx.doi.org/10.1590/s1982-21702017000300034

HUNG, C.; BRYSON, M.; SUKKARIEH, S. Multi-class predictive template for tree crown detection. Journal of Photogrammetry and Remote Sensing, v. 68, p.170183, 2012. https://doi.org/10.1016/j.isprsjprs.2012.01.009

INOUE, T., NAGAI, S., YAMASHITA, S., FADAEI, H., ISHII, R., OKABE, K., ... SUZUKI, R. Unmanned aerial survey of fallen trees in a deciduous broadleaved forest in eastern Japan. PLoS one, v. 9, n. 10, p. e109881, 2014. https://doi.org/10.1371/journal.pone.0109881

JAAKKOLA, A.; HYYPPÄ, J; KUKKO, A.; YU, X.; KAARTINEN, H.; LEHTOMÄKI, M.; LIN, Y. A low-cost multi-sensoral mobile mapping system and its feasibility for tree measurements. ISPRS journal of Photogrammetry and Remote Sensing, 65(6), 514-522, 2010.

$\mathrm{KOH}$, L.P.; WICH, S.A. Dawn of drone ecology: low-cost autonomous aerial vehicles for conservation. Tropical Conservation Science, v. 5, n. 2, p. 121-132, 2012.

LISEIN, J; $\quad$ PIERROT-DESEILLIGNY, M; BONNET, S; LEJEUNE P.A photogrammetric workflow for the creation of a forest canopy height model from small unmanned aerial system imagery Forests, 4, pp. 922-944, 2013. doi:10.3390/f4040922

MEDEIROS, F. A. Desenvolvimento de um veículo aéreo não tripulado para aplicação em agricultura de precisão. 2007. 102 f. Dissertação (Mestrado em Engenharia Agrícola)- Universidade Federal de Santa Maria, Santa Maria, 2007.

MICHEZ, A., PIÉGAY, H., JONATHAN, L., CLAESSENS, H., \& LEJEUNE, P. Mapping of riparian invasive species with supervised classification of Unmanned Aerial System (UAS) imagery. International Journal of Applied Earth Observation and Geoinformation, v. 44, p. 88-94, 2016 https://doi.org/10.1016/j.jag.2015.06.014

PANEQUE -GÁLVEZ, J.; MCCALL, M.K.; NAPOLETANO, B.M.; WICH, S.A.; KOH, L.P. Small drones for community-based forest monitoring: an assessment of their feasibility and potential in tropical areas. Forests, 5: 1481-1507, 2014. doi:10.3390/f5061481

PULITI, S.; ENE, L.T.; GAOBAKKEN, T.; NAESSET, E. Use of partial-coverage UAV data in sampling for large scale forest inventories. Remote Sensing of Environment, v.194, p.115-126, 2017. https://doi.org/10.1016/j.rse.2017.03.019 
ROKHMANA, C.A. The potential of UAV-based remote sensing for supporting precision agriculture in Indonesia. Procedia Environmental Sciences, V. 24 , P. 245 - 253, 2015. https://doi.org/10.1016/j.proenv.2015.03.032

RUZA, M.R.; DALLA CORTE; A.P.; HENTZ, A.M.K.; SANQUETTA, C.R.; SILVA, C.A.; SCHOENINGER, E.R. Inventário de Sobrevivência de povoamento de Eucalyptus com uso de Redes Neurais Artificiais em Fotografias obtidas por VANTs. Advances in Forestry Science, V.4, N.1, 2017.

UBIALLI, J. A.; FIGUEIREDO FILHO, A.; MACHADO, S. A.; ARCE, J. E. Comparação de métodos e processos de amostragem para estudos fitossociológicos em uma Floresta Ecotonal na região norte Matogrossense. Floresta, v. 39, p. 511-523, 2009.

VERGANI, A.R.; Sobrevivência de brotação de Eucalipto e delimitação da área de talhões pós-colheita utilizando Veículos Aéreos Não Tripulados. Trabalho de Conclusão de Curso (Especialização em Gestão Florestal) - Universidade Federal do Paraná, 2015.

WHITEHEAD, K; HUGENHOLTZ, C. H. Remote sensing of the environment with small unmanned aircraft systems (UASs), part 1: a review of progress and challenges. Journal of Unmanned Vehicle Systems, v. 2, n. 3, p. 69-85, 2014. doi:10.1139/juvs-2014-0007

WHITEHEAD, K., HUGENHOLTZ, C. H., MYSHAK, S., BROWN, O., Leclair, A., TAMMINGA, A., EATON, B. Remote sensing of the environment with small unmanned aircraft systems (UASs), part 2: scientific and commercial applications. Journal of unmanned vehicle systems, v. 2, n. 3, p. 86-102, 2014. doi:10.1139/juvs-2014-0006

ZHANG, J.; HU, J.; LIAN, J.; FAN, Z.; OUYANG, X.; YE, W. Seeing the forest from drones: testing the potential of lightweight drones as a tool for long-term forest monitoring. Biological conservation, v.198, p.60-69, 2016. https://doi.org/10.1016/j.biocon.2016.03.027 